\title{
EL DOBLE ACCESO FENOMENOLÓGICO-ÉTICO A LA REALIDAD DEL OTRO EN LA FILOSOFÍA DE PAUL RICOEUR
}

\section{Martín Grassi*}

Resumen: Este trabajo pretende introducir al lector en la problemática de la intersubjetividad desde la perspectiva de Paul Ricoeur, considerando el doble acceso, ético y gnoseológico, a la realidad del otro. Se estudiará en primer lugar cómo, a los ojos de Ricouer, la fenomenología husserliana debe enfrentar su prueba más definitoria, la de la explicitación del fenómeno de la intersubjetividad, y cómo puede superarla. En segundo lugar, se mostrará cómo Ricoeur no reduce el acceso al otro a la constitución egológica, sino que, por el contrario, la subordina al a priori ético del respeto, que pone al otro como otro antes de cualquier tipo de constitución fenomenológica o de aproximación simpática. Es nuestra intención mostrar que no se trata en Ricouer de excluir ninguno de los dos accesos, sino que se trata de una fenomenología genética que rastrea lo extraño desde lo propio, atendiendo ante todo a la intencionalidad ética del hombre como lo definitorio en el orden intersubjetivo.

Palabras clave: Ricouer, Intersubjetividad, Fenomenología Hermenéutica, gnoseología, ética

Abstract: This paper pretends to be an introduction to the problem of intersubjectivity from Paul Ricouer's point of view, considering the double access, ethical and gnoseological, to otherness reality. First, it will be examined how, according to Ricoeur, husserlian phenomenology must face its more definitory test, the explicitation of intersubjectivity phenomenon, and how can it be surpassed. Second, it will be shown that Ricoeur does not reduce the access to the other to egological constitution, but, on the contrary, subordinates it to the ethical a priori of respect, which poses the other as other before any attempt of phenomenological constitution or sympathetic approach. Our intention is to state

* Profesor y Licenciado en Filosofía por la Universidad Católica Argentina. Becario Doctoral del CONICET. Profesor adjunto en la Facultad de Filosofía de la Universidad del Salvador (Área San Miguel, Colegio Máximo). Profesor adscripto de en la Facultad de Filosofía y Letras de la UCA. E-mail: martingrassi83@gmail.com 
that Ricoeur does not exclude any of these two approaches: Ricouer's position is one of a genetical phenomenology which tracks foreign from owness, attending principally to the ethical intentionality of man as definitory in the intersubjective order.

Key words: Ricoeur, Intersubjectivity, Hermeneutical phenomenology, Theory of Knowledge, Ethics

\section{Introducción}

La cuestión de la intersubjetividad es una de las principales cuestiones a las que tiene que hacer frente la filosofía en orden a una comprensión más profunda de la existencia humana. La concepción de un hombre como un individuo aislado y solitario, autárquico y autónomo, es tan abstracta como la de un hombre sin carne y sin mundo. La evidencia del fenómeno intersubjetivo -es decir, la necesidad del otro para la propia existencia en los más diversos órdenes de la vida- reclama a la filosofía el reflexionar acerca de los alcances y las dimensiones ontológicas del ser-con propio del hombre, así como también de la condición de posibilidad misma de un reconocimiento de la subjetividad de un otro. A pesar de la importancia del fenómeno social y ético en la historia del pensamiento occidental, la cuestión de la intersubjetividad adquiere una densidad especial en la segunda mitad del siglo XIX y en la totalidad del siglo XX. Ya no sólo el hombre vive junto a otros hombres y precisa de ellos para una efectiva subsistencia, sino que el mundo y el hombre mismo se constituyen intersubjetivamente: el mundo deja de ser considerado como mera naturaleza para ser comprendido como cultura, y ya no se piensa al hombre como una sustancia constituida por sí misma, sino como una existencia histórica que se realiza en su ser (ya no sólo en su vivir) gracias a su participación en una comunidad que lo reconoce como tal. La diversidad de enfoques y de valoraciones de este fenómeno son signos de su complejidad y riqueza.

Entre las muchas aproximaciones a la dimensión intersubjetiva de la existencia, podemos detectar una de capital importancia: la de una fenomenología hermenéutica. Esta aproximación, de la cual trataremos en este estudio a partir de uno de los principales pensadores de esta corriente, 
Paul Ricoeur, ha enfrentado la cuestión de la intersubjetividad atendiendo a los diversos modos en que este fenómeno se presenta a la reflexión filosófica: desde el modo en que este fenómeno es aprehendido como tal hasta el modo en que se inserta en los horizontes históricos y culturales de la existencia misma y los define. El presente trabajo se inscribe en un proyecto más abarcativo, del cual sería ésta su primera parte, que pretende tan sólo ser una introducción al modo en que Ricouer ha asediado este fenómeno para rendir toda su significación gnoseológica, ética y existencial. Esperamos, a partir de los horizontes de sentido vislumbrados por el pensador francés, poder encaminarnos a una comprensión más cabal del fenómeno de la intersubjetividad.

\section{La constitución egológica del alter ego}

Entre las obras de Edmund Husserl, Meditaciones Cartesianas ha sido una de las más comentadas y ha adquirido en la escuela fenomenológica una importancia singular, entre otras cosas, por la cuestión tratada en la Quinta Meditación: el problema de la intersubjetividad, es decir, el problema de la constitución del alter ego a partir del ego. Paul Ricoeur le ha dedicado varias páginas y es infaltable en sus tratamientos de la intersubjetividad la referencia a esta obra de su maestro. Será parte de nuestra tarea mostrar en qué sentido Ricoeur se aleja y en qué sentido parece profundizar la propuesta husserliana.

La Quinta Meditación intenta dar respuesta a una objeción que parece salirle al encuentro a Husserl luego de sus resultados contenidos en las cuatro meditaciones que le anteceden: la fenomenología es una filosofía solipsista, en tanto que re-conduce el fenómeno a la conciencia constituyente. Este ataque no es vano ni fuera de lugar, puesto que la reducción a la esfera de lo propio y la reducción trascendental parecen dejar de lado la alteridad de la realidad -o la realidad de la alteridad- para llevarla al seno mismo de la conciencia ${ }^{1}$. Aun cuando la fenomenología

\footnotetext{
1 "Cuando yo, el yo que medita, me reduzco mediante la epocbé fenomenológica a mi ego trascendental absoluto, ¿acaso no me he convertido en el solus ipse? ¿Y no permanezco siéndolo en tanto que, bajo el título de fenomenología practico
} 
husserliana tome como piedra angular el Ego del ego cogito cogitatum -razón por la cual el descubrimiento cartesiano es también el punto de partida de la fenomenología-, sin embargo, toda la filosofía husserliana es un intento hercúleo por escapar de un idealismo absoluto, del postulado de una conciencia "creadora". De aquí la importancia de la intencionalidad en tanto estructura originaria de la conciencia como "conciencia de...": la conciencia no se basta a sí misma en su ser nóesis, sino que se realiza en el encuentro con su polo noemático, razón por la cual se habla de una conciencia constituyente, y no creadora.

Restará, entonces, ver cómo hacer frente a esta objeción de solipsismo, indagando el modo en que se da la experiencia del otro-ya del alter ego, ya del mundo objetivo-, pues la cuestión estriba en la posibilidad de un conocimiento verdaderamente trascendente, es decir que traspase las fronteras propias de la conciencia reducida y que, por lo tanto, impida la identificación del conocimiento con la esfera del ego trascendental. Sin embargo, esta experiencia del otro no deja de ser la experiencia del ego trascendental, por lo cual el sentido del otro debe constituirse en el yo y a partir del yo. Como afirma Ricoeur, "toda la Quinta Meditación va a sufrir la tracción más extrema entre estas dos exigencias: constituir al otro en mí, y constituirlo como otro" 3 .

Fiel a los principios propios de la fenomenología, Husserl establece que el modo de darse óntico-noemático del otro será el hilo conductor

consecuente exposición de mí mismo? ¿No habría, pues, que estigmatizar de solipsismo trascendental a una fenomenología que quería resolver problemas del ser objetivo y presentarse ya como filosofía?" (Husserl, Edmund, Meditaciones Cartesianas, México, Fondo de Cultura Económica, 1996, \$42, p. 149).

2 "A cet égard, le problème d'autrui joue le même rôle que, chez Descartes, la véracité divine en tant qu'elle fonde toute vérité et toute réalité qui dépasse la simple réflexion du sujet sur lui-même" (Ricoeur, Paul, "La Cinquieme Méditation Cartésienne", en: $A$ la école de la phenomenologie, Paris, Vrin, 1986, p. 197).

${ }^{3}$ Ibid., p. 199. 
trascendental para la experiencia constitutiva de la experiencia de lo otro ${ }^{4}$. Esta experiencia del otro se presenta de un modo triplemente paradójico, según señala Ricoeur ${ }^{5}$, interpretando la descripción que de ella realiza Husserl. De un primer modo, experimento a los otros como siendo objetos psico-físicos en el mundo, por lo cual, por un lado, son objetos ante mi conciencia, y, por otro, por su ser psico-físico, son sujetos en sí mismos, ante los cuales yo mismo me presento como objeto de sus mundos de experiencia. De un segundo modo, el mismo mundo presenta una doble faceta en tanto que es el "mundo privado" propio de la experiencia de la conciencia, y en tanto que es un "mundo objetivo", es decir, accesible por igual a todos los sujetos. Por último, de un tercer modo, los objetos de predicados espirituales, los objetos culturales, constituidos en mí y a partir de mí, son tales originariamente por la constitución activa de sujetos "extraños", miembros de una comunidad cultural particular. Como el mismo Husserl advierte, lo que parecía un problema singular de la fenomenología, el de la teoría trascendental de la experiencia del otro, la llamada "endopatía", se extiende a la teoría trascendental del mundo objetivo y de los objetos culturales, en tanto que los co-fundamenta. De aquí que el título mismo de la Quinta Meditación: "En que la esfera trascendental del ser se revela como intersubjetividad monadológica".

Ricoeur subraya con fuerza que el problema del otro es, tal como lo vio su maestro, una piedra de escándalo para la fenomenología, en tanto

4 "Tengo que atenerme imperturbablemente a que todo sentido que tenga y pueda tener para mí cualquier ser, tanto por lo que hace a su esencia como por lo que hace a su existencia real efectiva, es sentido en $-o$ bien, a partir de- mi vida intencional, a partir de sus síntesis constitutivas, aclarándoseme y revelándoseme en los sistemas de verificación unánime. Se trata, pues, de crear el suelo desde el que responderá todas las preguntas imaginables que puedan tener sentido, e incluso de plantearlas y responderlas paso a paso; de comenzar con un desarrollo sistemático de la intencionalidad patente e implícita en la que se 'hace' para mí el ser de los otros y se me expone según su contenido legítimo" (Husserl, E., Op. Cit., $\$ 43$, p. 152).

${ }^{5}$ Ricoeur, "La Cinquieme Méditation Cartésienne”, pp. 199-200. 
atendamos al sujeto trascendental que constituye lo real en y a partir de él mismo. Pero la importancia de la apuesta a la constitución egológica del otro reside en que la reducción a lo propio y el análisis de las intencionalidades que constituyen la alteridad, lejos de encerrar la fenomenología en el solipsismo, le da su auténtico alcance. No debemos, dice Ricoeur, comprender esta reducción a la esfera de lo propio como si se tratase de una explicitación de una génesis temporal de la alteridad constituida (en tal caso, se trataría de una fenomenología más de cuño hegeliano). Más bien, hay, entre la experiencia de mí mismo -reducido a la esfera de lo propio- y la experiencia del otro, una "filiación de sentido". Es decir, el sentido de lo otro es tomado del sentido "yo", puesto que es necesario que para que el sentido alter ego pueda ser constituido, antes es preciso donar sentido al ego, a partir del cual se predica alter. Lo extraño es tal porque existe lo propio, y no a la inversa. Lejos de ser la disolución del otro en mí, la reducción a la esfera de lo propio deja traslucir la paradoja como paradoja, es decir, el hecho de que el otro se me da sin dárseme, que el otro es otro porque yo soy yo. Se trata de una experiencia de transgresión de la ipseidad propia y del reflejo de mí mismo en el otro. "Las dos palabras claves, transgresión (überschreitet) y reflejo (Spiegelung), son los dos testigos de esta paradoja del desgarramiento (arracbement) de otra existencia a mi existencia, en el momento mismo en que pongo a ésta como única"'.

6 "La Cinquieme Méditation Cartésienne", p. 201. Luego de un análisis del tema de la analogía y de la intersubjetividad en los escritos inéditos de Husserl anteriores a las Meditaciones cartesianas, y proponiendo un paralelismo entre la analogía de Husserl respecto a este tema y la de Aristóteles respecto al ser, Ricoeur afirma: "Between the univocity of solipsism and the dissemination of totally different subjects there lies the narrow path of analogy, that is the analogy of the ego in the alter ego. It is in this way that Husserl invents anew -probably without knowing it- the analogy of being in the form of what could be called the analogy of the 'I am' " (Ricoeur, Paul. "Analogy and Intersubjectivity in Husserl, based upon previously unpublished writings from the period 1905-1920 (Edited by Iso Kern, Husserliana, vol XIII, Nijhoff, 1973)", documento inédito, Vansina: (1975) II.A.314, pág. 7-8). 
Esta reducción a lo propio, explica Ricoeur, es de por sí una abstracción, pues el hombre no existe de ningún modo solo, pero obedece a una necesidad metódica que permite poner en orden un sentido primero de ego y un sentido segundo, ateniente a todo lo que sea predicado como "otro". El producto de esta abstracción, por otro lado, es la unidad concreta cuyo núcleo es la "carne", el cuerpo vivo propio, a partir del cual se constituye una naturaleza primordial. Y es esta esfera de pertenencia de esta naturaleza primordial la que hace de fundación originaria (Urstiftung) a la cual remite todo estrato; en este sentido tal experiencia no está dada, sino que se trata de un anterior jamás donado y, sin embargo, encontrado a partir de una "depuración" (Reinigung) de todo aquello que no es lo propio, por lo cual, a pesar de su núcleo empírico, esta experiencia se mantiene en una interpretación o exégesis (Auslegung). Así, esta esfera primordial es suelo previo de toda constitución, término de una depuración, y punto de partida de un trabajo de constitución. Es un pre-dado que tiene la densidad propia de un yo soy que precede siempre al yo pienso. Esta toma de conciencia de lo propio se hunde así en una riqueza que excede toda reflexión. "Por un asombroso rodeo, el trascendental reducido revela lo óntico que desborda"7.

Este primer movimiento de la reflexión fenomenológica nos entrega el sentido de la encarnación, de una naturaleza mía, centrada sobre mi carne, con sus potencialidades y horizontes. Nos encontramos así con la plenitud del ego, en tanto polo de sus actos, que deviene babitus y luego mónada. A la vez, el mundo deja de ser mero espectáculo para devenir ambiente o mundo en torno (environnement) de mi carne. De esta dialéctica de lo "fuera de mí" y de lo "en mí" instituido por mi carne, procede toda la constitución de lo extraño "en" lo propio y "fuera" de lo propio.

La cuestión está entonces en interrogar la experiencia de lo otro, que parece presentar una trascendencia de grado superior a la "trascendencia inmanente" puesta de relieve en la reducción a lo propio. El tema decisivo de la "Quinta Meditación” es, entonces, para Ricoeur, el de la captación analogizante del otro como otro yo, por la cual se

7 “La Cinquieme Méditation Cartésienne”, p. 204. 
equilibran las dos exigencias de la fenomenología de respetar la alteridad del otro al tiempo que dicha experiencia de alteridad se enraíza en la esfera primordial, venciendo el solipsismo sin sacrificar la egología 8 .

Si el otro fuera simplemente otro, sería inaccesible y no habría intersubjetividad. Aunque si el otro fuera accesible por medio de una suerte de intuición -intuición emocional, por ejemplo- sería una mera extensión de mí mismo. En el primer caso, el sentido de "yo" se aplicaría de modo puramente equívoco; en el segundo caso, se aplicaría de manera unívoca. La solución no yace ni en la equivocidad del término "yo", ni en su univocidad, sino en la transferencia analógica desde lo propio bacia lo extraño, en cuanto que no hay ni reducción a lo mismo ni irreductibilidad de lo otro?.

Por un lado, la experiencia del otro se anuncia por su cuerpo físico presentificado, apresentando el ego que habitándolo lo hace ser cuerpo vivo $^{10}$. Por otro lado, gracias al entrelazo -en palabras de Merleau-Pontyentre mi carne y el mundo objetivo, me apercibo como realidad intramundana. La articulación entre la auto-percepción de mí mismo como ente mundano y la percepción del otro gracias a su cuerpo se da, según Ricoeur, en tres niveles. El primero es que la significación de mi cuerpo como cuerpo vivo se transfiere al cuerpo del otro gracias a una analogía que opera de carne a carne (en este punto, Ricoeur señala que se trata del texto más importante de la Quinta Meditación), una analogía que es pre-reflexiva, ante-predicativa, en el orden de las síntesis pasivas. Lo que especifica esta analogía es la llamada "parificación" (Paarung), la cual no va de objeto a objeto (como en todas las otras analogías), sino de lo propio a lo extraño, que realiza la transgresión de lo originario a lo no-originario,

${ }^{8}$ Cf. Ibid., p. 206.

9 Ricoeur, "Analogy and Intersubjectivity in Husserl", p. 2.

10 "C'est donc du côte du corps d'autrui qu'il faut chercher la clé du problème: lui seul peut à la fois m'être donné à percevoir tout en me donnant autrui" ("La Cinquieme Méditation Cartésienne", p. 206). 
gracias a semejanzas carnales ${ }^{11}$. La parificación suministra los elementos asociativos de la analogía, reconociendo en el otro la analogía de mi propia reificación o mundanización, y transfiriendo el sentido ego dado originariamente en la auto-percepción de mí mismo, al alter ego, cuyo ser sujeto nunca me será dado en forma originaria, en percepción impletiva.

El segundo nivel de articulación se da en el terreno de la verificación o confirmación del otro. En este punto, la verificación se realiza desde la concordancia de los comportamientos que indican, como signos, la vida que los anima, donando así una "accesibilidad confirmable" a esto que es "originariamente inaccesible", por lo cual la concordancia y la analogía no se contraponen.

En el tercer nivel, mientras que los análisis precedentes se movían en el círculo de la percepción, entra en juego la imaginación, el de las "variaciones libres" del aquí y del alli. La parificación parece menos enigmática, pues no sólo puedo acceder al otro por mi experiencia efectiva de la percepción, sino por mi experiencia potencial, imaginando que ocupa el allí que es aquí para el alter ego, recibiendo la apercepción analogizante un sentido concreto. "Esto que no era inicialmente más que una especie de la analogía lógica, así pues una especie de forma de duplicación, deviene ahora trasposición en imaginación y simpatía a otra vida"12.

De este modo, hay una progresión a la donación del otro en su verdad: en el primer nivel, tiene la forma de una ley universal, la parificación; en el segundo, un medio de seguridad o verificación; en el tercero, el contenido concreto en el modo "si yo estuviera allí". Sin

11 'Thus the absolutely primitive relation between what is my 'own' and what is 'foreign' is mastered conceptually, implying no chronological precedence and marking only the directional character of the relation on the transcendental relation. In order not to confuse this analogical relation with reasoning using the fourth proportional. Husserl prefers to compare it to 'association', that is to a relation belonging to the order of 'passive genesis'. Paarung operates in every experience directed from an original to something derived, from the same to the other" ("Analogy and Intersubjectivity in Husserl", p. 7).

12 "La Cinquieme Méditation Cartésienne", p. 211. 
embargo, debemos recordar, dice Ricoeur, que Husserl advierte sobre lo enigmático de esta última transferencia, puesto que nunca puedo introducir el allí del otro como mi aquí. Puede verse, así, que la exigencia idealista de la constitución, por la cual el otro debiera ser una modificación de mi mónada, se encuentra con la exigencia realista de la descripción, por la cual el otro no cesa de ser excluido de la esfera de lo propio. Y, por otra parte, debemos señalar junto con Ricoeur la subordinación de los modos de verificación al estatuto trascendental propio de la Paarung.

La experiencia actual y la experiencia potencial son los dos caminos que contribuyen a que la anticipación de una vida psíquica diferente a la mía -pero egológicamente como la mía- sea verificada. Pero ni percibir un comportamiento concordante ni imaginarme allí significaría que "alli" otro ego se posiciona él mismo para sí mismo, si la transferencia analógica de lo que es "lo propio" a lo que es "extraño" no gobernara trascendentalmente este modo dual de impleción ${ }^{13}$.

El problema ahora se presenta ante la constitución de una naturaleza en común y de un mundo cultural. La dificultad principal es cómo pasamos de la toma de conciencia del otro a la explicitación de la reciprocidad de los yoes. "La relación yo-otro es esencialmente asimétrica, no recíproca; la parificación está orientada del sentido único del ego primordial al ego análogo"14. La clave para esta cuestión será nuevamente la experiencia del otro en su cuerpo, en tanto que si el allí del otro es su aquí para sí mismo, entonces "mi" naturaleza es la misma que la del otro. La noción intermediaria es aquí la de perspectiva, por la cual la perspectiva originaria es la mía propia, mientras que las perspectivas de los "otros yoes" son apresentadas, en tanto se dirigen al mismo mundo y al mismo objeto. "Así, el idealismo monádico queda salvado en el realismo monadológico; es en el interior de mi esfera de pertenencia que se edifica

13 "Analogy and intersubjectivity in Husserl", p. 8.

14 “La Cinquieme Méditation Cartésienne", p. 212. 
el sentido de mundo percibido por un otro"15. Por otra parte, se agrega a esto la coordinación de las corrientes temporales de vivencias singulares en una única "comunidad temporal", siendo el tiempo la forma de coexistencia de la pluralidad de mónadas. Así como cualquier unidad constituida de forma intersubjetiva, también el tiempo objetivo del mundo es uno.

Por último, en lo referente a las comunidades intermonádicas, Ricoeur señala que las comunidades, en sentido estricto, son comunidades de personas a las que corresponden objetos culturales específicos. La constitución de un mundo común es el primer paso y el fundamento de toda comunidad intersubjetiva. A su parecer, la originalidad de Husserl reside justamente en su progresión metódica desde el solipsismo a la comunidad, fundando ésta última en la comunidad intencional de la cual la naturaleza objetiva es su correlato. Lo interesante en esta progresión es que hay en las comunidades históricas una igualación objetivante por la cual, así como el otro es tal para mí, yo soy otro para él, por lo cual puede hablarse de una comunidad de hombres reales. Aparece, así, la tensión entre la asimetría de la relación yo-otro -exigida por el idealismo monádico- y la igualación objetivante -exigida por el realismo sociológico-, paradoja que añade una dificultad más a la noción de intersubjetividad trascendental. Para resolverla, Husserl acude a la constitución de la psyché: la auto-objetivación de la mónada en psyché motiva y justifica el encuentro real entre los hombres en tanto que también yo soy otro para los otros, y que los otros son para mí, por lo cual la relación entre el yo y los otros es a un tiempo la de psiquismos recíprocos y separados.

Resta analizar la cuestión de las comunidades culturales y el modo en que éstas se relacionan con otros, con la misma dialéctica que animaba

15 Ibid., p. 215. A continuación, Ricoeur insiste sobre la unicidad del mundo objetivo: "Il y a bien alors deux couches, mais non deux mondes: une couche vécue en original, une autre apprésentée comme le sienne; (...) ces deux couches sont deux couches de l'objet, du même objet, dont je dis que je le perçois et que l'autre le perçoit aussi; mais cet 'aussi', qui procede du doublement de la conscience, ne fait pas un monde double". 
el caso singular entre lo propio y lo extraño. Se articula así la esfera del Espiritu, que se distingue de la psyché en tanto realidad natural (y aquí reconoce Ricoeur un vocabulario propiamente hegeliano y diltheyano). La persona no es sinónimo ni de bombre ni de ego, sino que es correlativa de la comunidad y de sus propiedades habituales, es decir, la persona es el ego considerado en sus habitualidades comunitarias. De esta forma aparece la noción de Lebenswelt o mundo de la vida -tan central a la última obra de Husserl, la Krisis-, que denota el polo opuesto a la constitución, el término al cual se orienta. En efecto, el mundo de la vida aparece como el mundo pre-dado, original, que es suelo (boden) de toda constitución, y en el cual se desarrolla la vida de la conciencia. Es un único mundo a la mano de todos, que se ofrece a todos por igual, y gracias al cual puede constituirse una verdadera intersubjetividad, una auténtica comunidad (aun las comunidades particulares pueden comunicarse entre sí y establecer comunidades más amplias gracias a este mismo suelo común), a la vez que posibilita una verdadera bistoria humana ${ }^{16}$.

Nos es imposible aquí detenernos en la caracterización y las implicancias de la noción husserliana del mundo de la vida, pero no pudimos dejar de señalar el papel central que ocupa para una consideración de la intersubjetividad. Volveremos sobre ella en la segunda parte, cuando abordemos propiamente la cuestión de la comunidad y de la dialéctica mutualidad-reciprocidad. Por ahora, el análisis se reduce a los modos de la constitución del otro desde el ego; el análisis del mundo de la vida, en cambio, implica ya la constitución efectiva del otro y los modos de relación entre las subjetividades en el marco de la comunidad. Antes, de todas maneras, deberemos hacer un rodeo por la constitución ética del alter ego para delimitar cuál es el papel, en rigor, del mundo de la vida en la

${ }^{16}$ Paul Ricoeur dedica dos artículos a la última obra sistemática de Husserl: "Lo originario y la pregunta-retrospectiva en la Krisis de Husserl" y "Husserl y el sentido de la historia". Ambos artículos se encuentran en versión castellana en: Acta fenomenológica latinoamericana, Bogotá, San Pablo, 2005, Vol. II. Puede consultarse también al respecto: Salinas Leal, Héctor Hernando, "Paul Ricoeur lector del último Husserl o las paradojas hermenéuticas de la Crisis", en: Acta fenomenológica latinoamericana, Bogotá, San Pablo, 2005, Vol. II, pp. 293-308. 
constitución de la intersubjetividad, pues aun cuando el mundo objetivo se constituya en un segundo momento a partir de la constitución de las conciencias ajenas, pareciera exigirse un mundo en común anterior a la misma constitución del otro que posibilite el reconocimiento mismo del otro como tal, con lo cual aparece el injerto hermenéutico en la fenomenología. Pero para poder desarrollar esta aparente paradoja, debemos examinar la dialéctica entre individuo y comunidad ${ }^{17}$, pasando por la ética del respeto como fundante de una fenomenología de la simpatía. No está de más señalar, sin embargo, que no hemos encontrado en los análisis de Ricoeur respecto al tratamiento de la intersubjetividad en Husserl un análisis de la noción de mundo de la vida, siendo el texto pivotante el de las Meditaciones cartesianas.

Esbozados los comentarios de Ricoeur sobre el texto husserliano, veamos ahora cuáles son sus conclusiones respecto a la posibilidad de la fenomenología como ciencia filosófica y su limitación en la cuestión de la intersubjetividad, como su más profunda paradoja, a la luz de las Meditaciones Cartesianas.

En primer lugar, en cuanto a la radicalidad del punto de partida exigida por la ciencia estricta que pretende ser la filosofía en el modo de la fenomenología, podemos ver en las Meditaciones Cartesianas una apuesta por el cogito cartesiano. Sin embargo, rompe luego con el sujeto impersonal propio de la herencia cartesiana y kantiana al referir todo sentido a una subjetividad singular y a una intersubjetividad monádica, anticipando así las reflexiones de la Krisis.

${ }^{17}$ En este sentido, la noción de Lebenswelt aparece anticipando los análisis de la Krisis en el marco de la constitución de las comunidades superiores. El punto tomará capital importancia en la dialéctica propia de los egos y las instituciones y los mundos culturales, abriendo a la fenomenología a los análisis sociológicos y contraponiéndola a la dialéctica en Hegel entre sujeto y espíritu objetivo (Cf. Ricoeur, Paul. "Hegel y Husserl sobre la intersubjetividad", en: Del texto a la acción: Ensayos de bermenéutica II, Buenos Aires, Fondo de Cultura Económica, 2006, pp. 259-278). 
En segundo lugar, en cuanto al método de explicitación (Auslegung) propio de la fenomenología, en las Meditaciones, el movimiento progresivo y sintético hacia lo concreto está subordinado al movimiento regresivo y analítico hacia lo original y radical. Así, la explicitación es una explicitación de la experiencia y no una construcción metafísica, por lo cual la fenomenología se ubica entre una filosofía de la construcción y una filosofía de la descripción, en un equilibrio entre el idealismo alemán y el empirismo inglés.

En tercer lugar, respecto a la sistematicidad propia de una ciencia filosófica, la fenomenología no puede ser sistemática al modo cartesiano de una construcción escalonada de verdades que va de una hacia otra y cuyo primer escalón fuese el cogito. Tampoco puede serlo al modo hegeliano. Hay en Husserl un proyecto de sistema, y es el de la estructura de la relación intermonádica de la que procede la necesidad del orden objetivo, por lo cual las infinitas libres combinaciones intersubjetivas se dan en un marco de un sistema de incomposibles aprióricos, al modo leibniziano. "Así, el solo sistema que la fenomenología puede concebir es un sistema de composibilidades, es decir, finalmente, de posibilidades; este sistema deja abiertas todas las cuestiones últimas. No es precisamente más que un sistema de 'sentido posible para nosotros' "18. Se entiende de este modo que la filosofía tenga la pretensión de una toma de conciencia universal (universale Selbstbesinnung), cuyo carácter universal es exigencia de su ser ciencia, a la vez que la toma de conciencia refiere a la posibilidad de cada quien a volver sobre sí en la reflexión y encontrar -al modo de Sócrates y de Agustín de Hipona, como cita Husserl en su página final de las Meditaciones- la verdad dentro de sí.

Como afirmamos al principio, y como Husserl y Ricoeur han visto bien, la cuestión de la intersubjetividad no es solo un problema más que la fenomenología deba atender, sino más bien, la piedra de toque fundamental de la fenomenología, su mayor desafío y su mayor hallazgo. Resta, ahora, estudiar el modo en que estas reflexiones toman cuerpo y

18 “La Cinquieme Méditation Cartésienne”, p. 224. 
encuentran un desarrollo dentro de la meditación de Ricoeur respecto de la esencia intersubjetiva de la persona humana.

\section{La constitución ética del prójimo}

La fenomenología como ciencia filosófica estricta ha debido superar la cuestión de la alteridad como su prueba más definitoria. Hemos visto cómo Husserl, sin ignorar ni la exigencia egológica de la constitución ni la exigencia realista de la descripción, ha propuesto las líneas de investigación y de respuesta a la pregunta por la constitución del alter ego. A su vez, hemos visto la evaluación de esta meditación por parte de Paul Ricoeur y el modo en que él entiende que la fenomenología cumple con los requisitos para ser una ciencia. Esto último no ha sido banal, puesto que el mismo Ricoeur se alista en las líneas de la fenomenología -si bien la enriquece con la perspectiva hermenéutica ${ }^{19}$ - y este balance de la Quinta Meditación valida de algún modo el método fenomenológico como acceso genuino a la verdad filosófica. Sin embargo, la cuestión de la intersubjetividad no se agota en la explicitación de la intencionalidad propia de la constitución del alter ego. Por el contrario, se trata tan sólo de un aspecto de la cuestión: se trata del aspecto gnoseológico del problema de la intersubjetividad. Pero, ¿es acaso el único modo de acceder al otro yo esta conciencia constituyente? ¿Puede hallarse otro acceso más allá del gnoseológico? ¿No es, en efecto, limitado y deficiente este camino fenomenológico? ¿En qué sentido un acceso distinto es imprescindible para la cuestión del otro yo? ¿Acaso el otro se me presenta tan sólo como un objeto del mundo que apresenta su ser sujeto? ¿No se presenta de otro modo por el cual yo me convierto no sólo en su conocedor o espectador, sino en su hermano o su custodio? En este apartado examinaremos cómo Paul Ricoeur emprende una crítica del camino fenomenológicognoseológico y propone como acceso pleno al alter ego el camino ético. Pero advirtamos desde ya que Ricoeur se caracteriza por un afán de

${ }^{19}$ Cf. Ricouer, P., "Fenomenología y hermenéutica: desde Husserl”, en: Del texto a la acción: Ensayos de hermenéutica II, Buenos Aires, Fondo de Cultura Económica, pp. 39-70. 
inclusión de todo punto de vista válido, por lo cual en el próximo apartado nos encontraremos con una revalidación del planteo husserliano, enriquecido por el momento ético que ahora desarrollaremos.

La cuestión de la intersubjetividad, tal como la ha planteado Husserl, presenta para Ricoeur, a pesar de su relevancia y de su invalorable aporte, dificultades y límites que han de superarse en orden a alcanzar la realidad del alter ego, que ahora tomará el nombre, menos abstracto y más apelativo, de tú. En otras palabras, ya no se tratará aquí del ser del alter ego como sujeto trascendental al modo como yo mismo, sino que se trata de la persona, de ese otro yo que es más que un mero sujeto apresentado y que forma conmigo una comunidad de amor. De hecho, Ricoeur ha tenido en su filosofía una impronta personalista muy fuerte, debido sobre todo a las enseñanzas de Gabriel Marcel y de Emmanuel Mounier, junto a la no menos importante de Karl Jaspers.

En uno de sus escritos tempranos ${ }^{20}$, Ricoeur define a la persona contraponiéndola al individuo, entendido como el temperamento prolongado por el carácter y coronado por la "mentalidad" (el se de su medio social, económico y cultural). De este modo, mientras que el individuo pasa a ser objeto de ciencia, la persona es, por definición, noobjetiva, y, por tanto, escapa al saber científico. Más bien pareciera que la clave para acceder a la realidad personal es la noción de acto:

¿Qué es, pues, la persona? Soy persona cuando YO hago lo que yo hago, es decir, en el sentido radical y radicalmente activo de la palabra "hacer", cuando aquello que hago se explica no por todas las fuerzas determinadas, sino por mi, por mi libre decisión. La persona actúa (agit) y no es actuada (agie). La noción de acto es evidentemente una clave de la idea de persona. La persona es quien reivindica un cierto acto, quien se solidariza con este acto, asumiendo las consecuencias, siendo responsable ${ }^{21}$.

${ }^{20}$ Ricoeur, P., "Note sur la personne”, en: Le Semeur, N7, Mai 1936, pp. 437-444, (Vansina: (1936) II.A.2a).

21 Ibid., p. 439. 
Así como el acto nunca es un acto de nadie, sino un acto tuyo o mío, así tampoco la persona no es nunca un él, sino un yo o un tú. De aquí que la persona y el acto sean irreductibles a la objetividad, puesto que ésta se define en su lejanía, en su tomar distancia, en su ser término del cual hablo, en tercera persona, y nunca aquello a lo cual hablo22. Claro que la encarnación como tal es la paradoja central de la persona, en tanto que la persona es objetivable gracias a su presencia mundana como cuerpo y está sujeta a las determinaciones del mundo en el que vive. Pero la paradoja, lejos de evitarse, es el único modo auténtico de acercarse a lo personal. Lo importante es que la persona es tal por una decisión existencial: o bien me realizo libremente o me someto a la esclavitud de las leyes naturales, sociales, económicas, etc. Así, el hombre es personal en tanto que impide que las ciencias del hombre sean rigurosas ${ }^{23}$.

La persona se manifiesta, entonces, esencialmente por una vocación de la cual es responsable, debiendo encarnarla en actos, los cuales serán entonces testimonio de dicho llamado. La realización suprema de esta responsabilidad es el compromiso, cuyo máximo cumplimiento se da en el sacrificio, que es la antítesis del suicidio, si bien es indiscernible de él en el plano meramente observacional. De este modo, la persona es tal por estar llamada por el Supremo Concreto, la Persona en sí, que es para el cristiano el mismo Cristo. De aquí que afirme que "yo soy más personal cuanto menos pienso en el ser" 24 . Sin poder detenernos en estos conceptos de vocación, responsabilidad, testimonio, etc., queremos tan solo señalar que la realidad personal se define en el orden de una libertad comprometida y amenazada siempre por la sombra de la traición a sí.

Podemos ver cómo ya en los principios del filosofar de Ricoeur se encuentra lo que luego desarrollará como la fenomenología del "hombre

22 La escisión entre lo objetivo y lo personal, y la subsiguiente crítica del objetivismo era un lugar común en la filosofía de principios de siglo pasado, sobre todo en las corrientes existenciales y personalistas. Un texto paradigmático en este sentido es el de Gabriel Marcel, "Existence et objectivité", Journal Métapbysique, Paris, Gallimard, 1935.

${ }^{23}$ Cf. "Note sur la personne", pp. 440-441.

${ }^{24}$ Ibid., p. 444. 
capaz", contenida sobre todo en sus trabajos Le volontaire et l'involontaire y en Soi-niêne comme un autre. En especial, la última aclaración del texto respecto a la persona me parece de singular importancia: "soy más personal cuanto menos pienso en el ser". Creo que debemos entender aquí por "ser" el modo en que lo entendían las filosofías idealistas, las cuales parecían identificar ser y objeto. En el siglo XX no será extraño este trascender el planteo del ser por el pensamiento, o más bien, el trascender la identidad ser-ente, como viera Heidegger. La persona es lo "más allá del ser", o lo "otro modo de ser", como planteará luego Emmanuel Levinas. En este sentido, pareciera que Husserl mismo, al pensar la intersubjetividad, hace de la persona un ser (en el sentido señalado), por lo cual sería necesario tomar cierta distancia respecto de su planteamiento para alcanzar el núcleo mismo de lo inter-subjetivo y de lo personal mismo.

La dimensión práctica de la ética parece tomar aquí la posición nuclear. En un artículo del año $1954^{25}$, Ricoeur muestra cómo el aparecer del otro supone una fundación en la posición de ser que excede todo método descriptivo y apunta, más bien, a una función práctica de la conciencia. Así, se traza un camino desde la fenomenología de Husserl y Scheler a la ética kantiana, desde la fenomenología de la simpatía a la ética del respeto. En última instancia, la posición absoluta del otro en el respeto fundamenta el modo de aparecer del otro, fundando así la fenomenología de la simpatía en la ética del respeto.

La fenomenología, en cualquier caso, es para Ricoeur decepcionante respecto al problema del otro yo, puesto que pone el aparecer del otro al modo del aparecer de las cosas, y así no resuelve el modo en que aparece, justamente, el otro, mi semejante. El doble enigma de la subjetividad, a la vez extraña y semejante, queda sin resolver por la "dictateur de la représentation", pues la presencia del otro, su posición absoluta queda "suspendida" por la epoché, tal como la emprende Husserl

${ }^{25}$ Ricoeur, Paul. "Sympathie et Respect: Phénoménologie et étique de la seconde personne", en Revue de Métaphysique et de Morale, vol. 59 (1954), N 4, pp. 380-397. 
en la Quinta Meditación: la reducción a la esfera de lo propio ${ }^{26}$. En efecto, la epoché a la esfera propia es precaria en tanto que la constitución del otro por la descripción pura y simple de características propias que lo excluyen de mi esfera propia no es suficiente para que el "valor ontológico" del otro sea distinto al de las cosas.

Tampoco el intento de Max Scheler -en su obra Esencia y formas de la Simpatía - parece ser suficiente para Ricoeur. Scheler intenta acceder a la revelación de la existencia del otro no ya desde una perspectiva teorética, sino afectiva, a través de la simpatía, entendida como una manera de "tomar parte" de una alegría o tristeza ajena, sin reduplicarla de hecho. El problema para Ricoeur es que no se puede establecer una "distancia fenomenológica" entre los seres, entre yo y el otro, a partir de un tratamiento descriptivo elevado a rango de esencia o de a priori. Por otra parte, no hay razón para privilegiar la simpatía por sobre los otros sentimientos intersubjetivos, ni desprenderse tampoco del orden descriptivo. ¿Por qué no establecer el momento negativo visto por Hegel, Marx y Sartre como aquél que se encuentra en el corazón de las relaciones interhumanas? Tienta la idea de pensar que la "oposición" de las conciencias es la clave de su "alteridad".

Para Ricoeur, Kant ha visto muy bien la correlación entre obligación y existencia del otro: "El otro es un entre (entre) de obligaciones para mí, y la obligación es un compendio (abrégé) abstracto de comportamientos posibles respecto al otro"27. Así, mientras que el cogito cartesiano es indiferente éticamente respecto al otro, la posición del otro en tanto que otro, el reconocimiento de una pluralidad y de una alteridad mutua no puede ser sino ética. Si bien Kant no tematiza explícitamente la cuestión del otro como tal, es en el análisis del respeto en el que se encuentra la clave kantiana de la existencia del otro. En la segunda formulación del imperativo categórico se introduce la noción de persona como aquello que se posiciona ante mí con una voluntad propia y, por tanto, limitándola, pues no puedo hacer uso de la persona como medio

26 “On peut dire que la V Meditation est une gageure intenable” (Ibid., p. 382).

27 Ibid., p. 388. 
para un determinado fin -como en el caso de las cosas- sino que la persona se presenta como un fin en sí mismo, y así estoy obligado a respetarla. El reconocimiento del otro es, entonces, correlativa a la doble instancia del deber ser y de la racionalidad práctica.

La existencia del otro es un postulado, un principio de orden existencial implicado en el comienzo de toda moralidad y por el cual se señala un reino de fines, es decir, un entramado sistemático de seres racionales por la ley misma del recíproco respeto. A este postulado no se accede por una reflexión acerca de los actos del cogito, sino por los análisis de las intenciones de la voluntad buena, que implica el acto de situarse a sí mismo en un todo de personas como miembro y soberano de la comunidad ética, regida por la reciprocidad del respeto.

El respeto opera la justificación crítica de la simpatía, en cuanto se dirige a la confusión afectiva inherente a ella. Sin embargo, no se debe escindir el respeto de la simpatía, como si el primero fuese trans-afectivo. El respeto remodela interiormente un afecto, lo cual implica que el respeto se presenta como "móvil a priori" frente a los móviles empíricos de la sensibilidad. Por lo tanto, respeto y simpatía no se contraponen, sino que son momentos de una misma dinámica vital.

La simpatía y el respeto son una y la misma "vida" (vécu): la simpatía es el respeto considerado en su materia afectiva, es decir, en su raíz de viviente, en su impulso (élan) y confusión; el respeto es la simpatía considerada en su forma práctica y ética, es decir, como posición activa de un otro Sí, de un alter ego ${ }^{28}$.

El respeto tiene, empero, como ya hemos señalado, una posición privilegiada frente a la simpatía, en tanto que la justifica, y esto de un doble modo. De un primer modo, elimina la equivocidad de la simpatía, manteniendo la alteridad de las personas que la fusión afectiva tiende a anular. De un segundo modo, el respeto justifica la simpatía al privilegiarla por sobre los otros afectos intersubjetivos, en tanto que su superioridad

${ }^{28}$ Ibid., pp. 391-392. 
existencial es consecuencia de su afinidad con la ética del respeto. En efecto, todo afecto tiene un momento de "toma de posición" frente al otro, un acto de apreciación, de evaluación de la existencia-valor del otro, por lo cual todo afecto intersubjetivo puede ser situado en relación al respeto.

La consonancia y la disonancia con el respeto, por consiguiente, forman parte de la estructura ética de todos los afectos intersubjetivos por medio de la "toma de posición" que los determina prácticamente; por el respeto el otro continúa siendo oscuramente reconocido, aun cuando sea apasionadamente suprimido y negado en intención o de hecho ${ }^{29}$.

La simpatía puede llamarse, frente a los otros afectos intersubjetivos, por su cercanía a la ética del respeto, "la splendeur du réspect" 30 , posicionándolo como un afecto purificador. De aquí que, en Scheler, tome la simpatía un lugar central en tanto que nos permite salir de la "ilusión timética", propia de la fenomenología. La obra de Scheler, en efecto, tiende a una concepción de la intuición que consiste, según Landsberge, en "rendir justicia" al valor de los seres.

Pero hay también un tercer modo en que el respeto justifica a la simpatía, y es en tanto que la coordina a la lucha y al conflicto interpersonal, y la subordina al respeto mismo. En efecto, el elemento negativo de la lucha aporta el momento dialéctico y el momento histórico a la ética del respeto y a la fenomenología de la simpatía. Pareciera que la simpatía permanece en el dominio privado de las relaciones humanas, mientras que la lucha parece habitar el plano de las fuerzas que mueven a la historia ${ }^{31}$.

\footnotetext{
29 Ibid., p. 393.

30 Ibid. Esta expresión nos recuerda a la referente a la belleza, que para los medievales era "splendor veritatis".

31 "La sympathie est sur la voie des relations anonymes aux relations personnelles; si la lutte 'historise', la sympathie 'intimise' les relations inter-humaines" (Ibid., p. 394). En una entrevista, Ricoeur entiende al personalismo justamente como la
} 
Es importante notar, señala Ricoeur, que la lucha no es lo originario ni lo puramente negativo. La lucha no tiene una estructura simple, por lo cual la fenomenología no puede tomarla como lo originario. Más bien es necesaria una labor analítica regresiva que permita explicitar las condiciones mismas de la lucha, descubriendo así el respeto implícito en ella y recomponiendo así su sentido. "Ésta sería la tarea de una genealogía de las pasiones, edificada sobre el fundamento del respeto; la lucha aparecería como una de las dramatizaciones pasionales de la revelación de la alteridad instituida por el respecto" 32 . De este modo, se subordina la lucha al respeto y la oposición a la alteridad, y dicha subordinación es tanto del orden teórico como del práctico. El respeto, entonces, no sólo justifica la simpatía como momento trans-afectivo, sino que también rectifica prácticamente la lucha, por lo cual se erige como momento transhistórico, que nos permite aceptar o rechazar lo que la historia produce en el dolor. El respeto dona así una medida a la violencia, anticipando el fin de la lucha en el doble sentido de misión moral y de término histórico. De aquí el invalorable testimonio concreto y actual de la no-violencia que invita a la fraternidad y amistad entre los hombres, mostrando así los fines humanistas de la historia, por la "fuerza desarmada" del respeto en medio de un mundo violento ${ }^{33}$.

Al final de este camino, Ricoeur pasa a las conclusiones metodológicas que de aquí se desprenden. En primer lugar, no se debe elegir entre una ética formal del respeto y una ética material de la simpatía o de la lucha, sino que el formalismo cierne a la ética de una armadura a

utopía de llevar al mundo de las relaciones anónimas y de las instituciones y sistemas, el encuentro de un yo y de un tú. Es la utopía de una sociedad que consista en relaciones de persona a persona, y, en tanto utopía, es el polo ideal al que debemos aspirar, aunque no sea posible alcanzar. Es el ideal de toda sociedad de personalizar cuanto sea posible las relaciones sociales (Cf. "Intersubjectivité, socialité, religion". Entrevista a Paul Ricoeur por Attilio Danese, en: Notes et documents, Dossier: Le retour de la philosophie pratique, (avril-juin 1986), p. 77).

32 Ricoeur, "Sympathie et Respect: Phénoménologie et étique de la seconde personne", p. 395.

${ }^{33}$ Ibid., p. 396. 
priori implicada en el momento de "toma de posición" respecto al otro, por lo cual no hay moralidad sin un momento formal ${ }^{34}$. Segundo, hay un reconocimiento del otro ligado al momento formal de la ética: la posición del otro implicada en la voluntad buena constituye el aspecto práctico y ético de la finitud. Tercero, la posición formal del otro en el respeto se logra doblemente en la simpatía y en la lucha, coordinándose así una fenomenología de la simpatía y una historia de la violencia. Por último, sin la posición de realidad correlativa al respeto, la fenomenología no puede pasar del problema de la constitución de la cosa al de la constitución de la persona. La persona es reconocida en el movimiento inverso al fenomenológico que reubica el aparecer en el ser. A su vez, esta posición de realidad de las personas tiene una dimensión de reconocimiento práctico, por la cual la existencia revela una existencia-valor. El deseo de conocer especulativamente la existencia del otro es ya indiscreción, falta de respeto: "poniendo la realidad del otro, el respeto la protege contra la vana curiosidad del saber"35.

\section{Conclusión}

El problema de la intersubjetividad ha marcado profundamente a la filosofía del siglo pasado, y ha tenido en el movimiento fenomenológico un lugar privilegiado. Hemos visto cómo Ricoeur retoma las reflexiones en torno a la constitución realizadas por Husserl, y también cómo aboga por un acceso ético a la realidad del otro yo, del prójimo, evaluando el acceso gnoseológico como impío en cuanto que pareciera reducir la alteridad del tú y así convertirlo en objeto de saber. En esta indecisión por un camino determinado que nos lleve al alter ego, o al tú, hay algo que se mantiene intacto en las reflexiones de Ricoeur: el carácter de absoluto que adquiere la persona que tengo frente a mí, y la imposibilidad de intromisión del sujeto que lo conoce. En este sentido, podemos ver una

\footnotetext{
34 Para un desarrollo de la propuesta ética, cf. Ricouer, Sí mismo como otro, Madrid, Siglo XXI, 2006, estudios VII-IX.

35 "Sympathie et Respect: Phénoménologie et étique de la seconde personne", p. 397.
} 
cierta resonancia con el planteo de Husserl, en tanto que la captación analógica apunta sobre todo a subrayar la independencia del alter ego respecto de $\mathrm{mi}$ propia conciencia, por lo cual no puede haber una presentación originaria del otro como tal. En este sentido, creemos que el planteo de Ricoeur respecto a la segunda persona, al tú, no sigue completamente al planteo radical de Emmanuel Lévinas, para quien el único acceso al otro es el acceso ético, con una predominancia de la alteridad sobre la mismidad. Ricoeur, por el contrario, parece buscar la génesis de sentido de lo extraño desde lo propio, por lo cual considera positivamente -aunque con reservas- el acceso gnoseológico propuesto por Husserl. A este acceso, Ricouer le añade el acceso ético, que, gracias al a priori del respeto, permite que el otro, que el prójimo, se me presente como un sujeto inalienable e inviolable, con una dignidad que regula todos mis actos libres. Queda por estudiar, en un segundo trabajo, cómo se articulan el plano gnoseológico y ético en el acceso al otro, a partir del desarrollo de una fenomenología del hombre capaz y de la estructura compleja del reconocimiento recíproco entre las personas.

Recibido 03/2011; aceptado 07/2011. 